\title{
Sparfloxacin in the treatment of drug resistant tuberculosis or intolerance of first line therapy
}

\author{
A. Lubasch*, R. Erbes*, H. Mauch*, H. Lode*
}

\begin{abstract}
Sparfloxacin in the treatment of drug resistant tuberculosis or intolerance of first line therapy. A. Lubasch, R. Erbes, H. Mauch, H. Lode. C) ERS Journals Ltd 2001.

ABSTRACT: Patients with multiresistant tuberculosis (TB) and patients with intolerance of first line antituberculosis drugs present a major treatment problem. Sparfloxacin is highly active against mycobacteria, but the use is restricted by side effects and the contribution to antituberculosis therapy is unclear. A prospective study has therefore been performed to analyse the efficacy and tolerability of sparfloxacin in cases of resistant TB or intolerance of first line therapy.

Between April 1993 and April 1999, 30 TB patients (28 with pulmonary TB and two with lymph node $\mathrm{TB}$ ) were treated with combinations of sparfloxacin and at least two other drugs at the Chest Hospital Heckeshorn, Berlin. Sixteen patients were infected by resistant mycobacteria (one single drug resistance (SDR), one polyresistance, and 14 multidrug resistances (MDR); 14 males (age range 23-53 yrs), 2 females (68-74 yrs)). Twelve patients (11 males, one female, $27-80 \mathrm{yrs}$ ) had not tolerated first line antituberculosis drugs. Two additional male patients had continuous proof of Mycobacterium tuberculosis in sputum without resistance during therapy

The duration of sparfloxacin therapy during hospitalization ranged 2.5-4 months. Twenty-five patients completed therapy and were cured according to this study's definition. Although sparfloxacin was generally well tolerated, five mild phototoxic reactions and six moderate prolongations of the electrocardiographic QT-interval (30$\mathbf{4 0} \mathbf{m s}$ compared to baseline $\leqslant \mathbf{4 5 0} \mathbf{m s}$ ) were registered without clinical symptoms in the patient group.

In summary, sparfloxacin proved an effective and safe alternative antituberculosis drug for complicated tuberculosis.

Eur Respir J 2001; 17: 641-646.
\end{abstract}

\author{
*Depts of Chest and Infectious Dis- \\ eases and ${ }^{\#}$ Microbiology, Chest Hospi- \\ tal Heckeshorn, Berlin, Germany. \\ Correspondence: A. Lubasch \\ Dept of Chest and Infectious Diseases \\ Chest Hospital Heckeshorn \\ Zum Heckeshorn 33 \\ D-14109, Berlin \\ Germany \\ Fax: 4903080022623
}

Keywords: Intolerance of antituberculosis therapy

multiresistant tuberculosis

sparfloxacin

Received: August 91999

Accepted after revision November 13 2000
The standard antituberculosis therapy in sputum smear positive cases is a combination of isoniazid (INH), rifampicin (RMP), pyrazinamide (PZA) and ethambutol (EMB) for 2 months, followed by INH and RMP for further 4 months [1]. In cases of sputum smear negative patients, a regimen of three drugs (INH, RMP and PZA for 2 months, INH and RMP for further 4 months) is recommended [1].

The global increase of multiresistant Mycobacterium tuberculosis strains [2-7] and intolerance of first line antituberculosis drugs (INH, RMP, PZA, EMB, streptomycin $(\mathrm{SM}))[8,9]$ may cause major treatment problems and necessitate modification of the standard therapy regimen.

Recently developed drugs like fluoroquinolones show sufficient antimycobacterial activity and may play a significant role in the treatment of MDR tuberculosis (TB) and in cases of severe intolerance of first line antituberculosis medication $[2,5,10,11]$.

Sparfloxacin currently seems to be the most potent fluoroquinolone against $M$. tuberculosis, including multiresistant strains [2,11-14], although it may show some problematic side effects (e.g. phototoxic reactions). However, as there were no reports on the use of sparfloxacin in patients with MDR TB or intolerance of first line antituberculosis drugs in the literature, a prospective study was performed to analyse sparfloxacin's efficacy and tolerability in such cases.

\section{Material and methods}

\section{Sparfloxacin}

Sparfloxacin is a highly potent fluoroquinolone against a wide range of gram-positive and gram-negative bacteria, anaerobes, Legionella spp., Mycoplasma spp., Chlamydia spp. and Mycobacteria spp., including multiresistant strains $[12,15,16]$. The elimination half-life $(\mathrm{t} 1 / 2)$ is approximately $15-20 \mathrm{~h}$ which allows once daily administration. Sparfloxacin diffuses well into tissues and respiratory tract secretion and concentrates within macrophages, where mycobacteria survive and multiply $[12,17]$.

Sparfloxacin is generally well tolerated. While gastrointestinal reactions are the most frequent side effect, photosensitivity reactions and cardiovascular tolerability (electrocardiograph QT-interval) should be given special attention $[12,17-22]$. Due to its phototoxic 
potential, the use of sparfloxacin should be limited to respiratory infections with resistant organisms.

In this study, sparfloxacin tablets were administered once daily (dosages: $\leqslant 50 \mathrm{~kg}$ body weight, $200 \mathrm{mg} ; 50$ $70 \mathrm{~kg}$ body weight, $300 \mathrm{mg}$; $>70 \mathrm{~kg}$ body weight, 400 $\mathrm{mg}$ ) under directly observed therapy (DOT).

\section{Study design}

In a prospective study, the data of 30 hospitalized TB patients treated with sparfloxacin at the Chest Hospital Heckeshorn, between April 1993 and April 1999 were analysed. The patients were divided into three groups: resistant group $(n=16)$, intolerance group $(n=$ 12) and continuous proof group $(n=2)$. Patient characteristics are listed in table 1.

\section{Definitions}

The following definitions were used [23]: mono or single drug resistance (SDR): resistance to a single drug; multi-drug resistant (MDR): resistance to at least INH and RMP; polyresistance: resistance to more than one drug excluding MDR.

Hepatic intolerance was defined as an increase in liver enzymes to more than three times the normal limit during therapy and a rapid new increase in liver enzymes after re-exposure. Evidence of considerable amounts of acid-fast bacteria in sputum for over 10 weeks was defined as continuous proof of $M$. tuberculosis. Patients consuming more than $80 \mathrm{~g}$ of alcohol per day were defined as alcohol abusers. The treatment outcome was assessed using standard categories [24]. A patient who had completed a full course of anti-TB therapy with documented conversion of culture during the continuation phase was defined as cured.

\section{Diagnostics}

Current standards were used in the diagnostic procedures for TB [25]. The most frequently investigated specimens were sputum. Specimens were also obtained via fibreoptic bronchoscopy or lymph node puncture. After acid-fast bacteria staining using the auramine fluorescence method [26], the obtained specimens were investigated by fluorescence microscopy. Primary cultures were grown in soluble media (Middelbrook 7H9 broth) and on Löwenstein-Jensen solid medium [26]. Susceptible testing was performed on LöwensteinJensen solid medium by the breakpoint technique using the standard proportion method [26]. Resistance was diagnosed when $<1 \%$ inhibition of the original inoculum of $M$. tuberculosis occurred on LöwensteinJensen medium containing the following concentrations of antibiotics: INH: $0.25 \mathrm{mg} \cdot \mathrm{L}^{-1}$; RMP: $32.0 \mathrm{mg} \cdot \mathrm{L}^{-1}$, PZA: $125 \mathrm{mg} \cdot \mathrm{L}^{-1}, \mathrm{EMB}: 1.0 \mathrm{mg} \cdot \mathrm{L}^{-1}, \mathrm{SM}: 4.0 \mathrm{mg} \cdot \mathrm{L}^{-1}$, PTH: $32.0 \mathrm{mg} \cdot \mathrm{L}^{-1}$ [27]. The minimal inhibition concentrations (MICs) of all first and second line antituberculosis drugs and sparfloxacin were determined from strains resistant to first line antituberculosis drugs. The breakpoints for susceptibility of sparfloxacin were $\leqslant 1 \mu \mathrm{g} \cdot \mathrm{mL}^{-1}$ : susceptible; $1-4 \mu \mathrm{g} \cdot \mathrm{mL}^{-1}$ : moderately susceptible; and $>4 \mu \mathrm{g} \cdot \mathrm{mL}^{-1}$ : resistant $[12,15]$.

Patient compliance in the continuous proof group was controlled by urine tests for INH.

\section{Follow up}

Specimens were obtained weekly from patients able to produce sputum during hospitalization. Those with resistant tuberculosis were isolated until sputum culture conversion. Weekly laboratory tests for hepatic, haematological or renal side effects were also performed. Chest radiographs were performed monthly until discharge from the hospital. The chest radiographs were assessed by two independent physicians (a radiologist and a pulmonologist). Electrocardiography (ECG) examinations for control of the QT-interval were done every two weeks, and twice weekly in cases of QT prolongation.

After discharge from the hospital, patients were treated on an outpatient basis by their pulmonologists.

Table 1. - Patient characteristics

\begin{tabular}{lcccc}
\hline & All patients & Resistance group & Intolerance group & Continuous proof group \\
\hline Patients n & 30 & 16 & 12 & 2 \\
Mean age yrs & 46.4 & 40.6 & 55 & 41.5 \\
Median (range) age yrs & $41(23-80)$ & $37.5(23-74)$ & $60(27-80)$ & $2 / 0$ \\
Sex male/female & $27 / 3$ & $14 / 2$ & $11 / 1$ & 2 \\
German-born & 12 & 2 & 8 & \\
Non-German-born & 18 & 14 & 4 & $0 / 2$ \\
Relapse & 17 & 13 & $4 / 6$ & 1 \\
Uni/bilateral disease & $9 / 19$ & $5 / 11$ & 6 & 2 \\
Cavitary disease & 15 & 11 & 2 & 2 \\
Lymph node TB & 2 & $15^{*}$ & 12 & 2 \\
Sputum smear positive & 20 & 1 & 4 & \\
Culture positive & $29^{*}$ & & & \\
Alcohol abuse & 7 & & & \\
i. . Drug abuse & & & & \\
HIV positive & & &
\end{tabular}

*: one patient was treated because of progression in chest radiography and history of multidrug-resistant tuberculosis (TB); \#: only two patients (aged 41 and 42, respectively). HIV: human immunodeficiency virus. 
The practitioners were contacted at the end of therapy to evaluate the treatment outcome.

\section{Results}

\section{Resistance group}

The group with resistant micro bacteria (16 patients) comprised 14 non-German born males with a mean age of 37 yrs (range 23-53 yrs) and 2 elderly German females $(68-74 \mathrm{yrs})$. Thirteen patients had a relapse of pulmonary tuberculosis. All patients had pulmonary TB ( 5 unilateral, 11 bilateral), and there were 8 cases with cavitation. Ten patients had positive sputum smears and 15 presented with positive cultures. One patient with a history of MDR TB had no proof of acid-fast bacteria in sputum smear and bronchoscopic specimen. Sputum culture, culture of bronchoscopic specimen and polymerase chain reaction (PCR) were also negative, but the patient was still treated because of progression in the chest radiograph.

One patient had SDR TB (INH), one was polyresistant $(\mathrm{INH}+\mathrm{SM})$, and 14 had MDR TB. Resistance details are listed in tables 2 and 3. All resistant and multiresistant $M$. tuberculosis strains were sensitive to sparfloxacin. The average MIC value was 0.4 $\mu \mathrm{g} \cdot \mathrm{mL}^{-1}\left(0.12-1.0 \mu \mathrm{g} \cdot \mathrm{mL}^{-1}\right)$ (breakpoint for susceptibility: $\left.\leqslant 1 \mu \mathrm{g} \cdot \mathrm{mL}^{-1}\right)$. The treatment regimens for the resistance group are shown in table 4 and the dosages and durations in table 5. Sputum cultures turned negative 2-24 weeks (median; 4 weeks) after start of treatment. No relapses were observed in these patients until discharge from the hospital. On discharge, 10 patients had significantly improved chest radiographs compared to the findings before treatment. Thirteen of the 16 patients completed therapy ( 24 months) and were cured according to this study's definition. One patient is still receiving treatment, while two could not be followed up after leaving Berlin to an unknown destination.

Table 2. - Singledrug-, polydrug- and multidrug-resistance in the 16 patients of the "resistance group"

\begin{tabular}{lccc}
\hline & $\begin{array}{c}\text { Single drug } \\
\text { resistance }\end{array}$ & Polyresistance & $\begin{array}{c}\text { Multidrug } \\
\text { resistance }\end{array}$ \\
\hline Patients n & 1 & 1 & 14 \\
INH & 1 & 1 & $14(100 \%)$ \\
RMP & & & $14(100 \%)$ \\
PZA & & $6(43 \%)$ \\
EMB & & $8(57 \%)$ \\
SM & & $9(64 \%)$ \\
Rifabutin & & & $12(86 \%)$ \\
PTH & & $1(7 \%)$ \\
CS & & $3(21 \%)$ \\
PAS & & & $1(7 \%)$ \\
SMZ & & & $3(21 \%)$ \\
AM & & & $1(7 \%)$ \\
\hline
\end{tabular}

INH: isoniazid; RMP: rifampicin; PZA: pyrazinamide; EMB: ethambutol; SM: streptomycin; PAS: para-aminosalicylic acid; PTH: prothionamide; CS: cycloserin; SMZ: Sulfamethoxazole; AM: amikacin.
Table 3. - Multidrug resistance (MDR) in 14 of the resistance group

\begin{tabular}{lcl}
\hline $\begin{array}{c}\text { Resistance } \\
\text { Number of } \\
\text { patients }\end{array}$ \\
\hline $3 \mathrm{x}$ & 3 & INH+RMP+Rifa \\
$4 \mathrm{x}$ & 1 & INH+RMP+EMB+SM \\
& 1 & INH+RMP+PZA+PAS* \\
$5 \mathrm{x}$ & 1 & INH+RMP+SM+Rifa \\
& 1 & INH+RMP+PZA+SM+Rifa \\
$6 \mathrm{x}$ & 1 & INH+RMP+EMB+Rifa+SMZ \\
& 1 & INH+RMP+EMB+SM+Rifa \\
& 1 & INH+RMP+PZA+EMB+SM+Rifa \\
$8 \mathrm{x}$ & 1 & $\begin{array}{c}\text { INH+RMP+PZA+EMB+Rifa+CS } \\
\text { Rifa+CS+SMZ }\end{array}$ \\
& 1 & $\begin{array}{c}\text { INH+RMP+PZA+EMB+SM+ } \\
\text { Rifa+PTH+AM }\end{array}$ \\
& 1 & INH+RMP+EMB+SM+Rifa+CS+ \\
& 1 & SMZ+CLM \\
\hline
\end{tabular}

*: patient with history of MDR tuberculosis. INH: isoniazid; RMP: rifampicin; PZA: pyrazinamide; SM: streptomycin; EMB: ethambutol; PAS: para-aminosalicylic acid; PTH: prothionamide; CS: cycloserin; Rifa: rifabutine; AM: amikacin; SMZ: Sulfamethoxazole.

\section{Intolerance group}

The intolerance group was comprised of 11 male patients and one female patient with a mean age of 55 (27-80 yrs). Eight patients were German-born, 10 patients had pulmonary (four unilateral, one with cavitary and 6 bilateral, one with cavitary) and 2 had lymph node TB. All 12 patients had positive cultures and seven were sputum smear positive. In four cases, a history of alcohol abuse was reported. Seven patients showed significant hepatic intolerance and three had severe skin reactions (exanthema) to first line antituberculosis drugs. INH and SM were contraindicated in one patient due to polyneuropathy and deafness, and one patient reacted with grand mal epilepsy to INH and with hepatic intolerance to RMP and PZA. All patients in this group were treated with combinations of three or four antituberculosis drugs, depending on individual intolerances, contraindications and sensitivity tests. Sputum culture conversion occurred after 3-12 weeks (median 6 weeks). All patients had significantly improved chest radiograph upon discharge from the hospital. Dosages and durations of sparfloxacin treatment during hospitalization are shown in table 5. All 12 patients could be defined as cured at the end of therapy (12 months).

\section{Continuous proof group}

The third group consisted of two German born male patients (aged 41 and 42 yrs) with continuous proof of $M$. tuberculosis strains (10 and 12 weeks, respectively) during therapy without demonstrated resistance. Compliance was controlled by proof of INH in urine. Both had bilateral pulmonary TB, one with cavitation, and both had a history of alcohol abuse. The sputum cultures turned negative 
Table 4. - Treatment regimens of the 16 patients in the resistance group (single-, poly- and multi-drug resistance)

\begin{tabular}{ll}
\hline Resistance & Treatment \\
\hline INH (+SM-CI) & RMP+PZA+EMB+SPFX \\
INH+SM (+PZA+RMP-INT) & EMB+PAS+PTH+TZ+SPFX \\
INH+RMP+Rifa (+PZA-INT) & EMB+SM+PAS+PTH+SPFX \\
INH+RMP+Rifa (+SM-CI) & PZA+EMB+PTH+SPFX \\
INH+RMP+Rifa (+PZA-INT) & EMB+SM+PTH+SPFX \\
INH+RMP+EMB+SM & PZA+PTH+PAS+CS+SMZ+SPFX \\
INH+RMP+PZA+PAS* & EMB+SM+PTH+SPFX* \\
INH+RMP+SM+Rifa & PZA+EMB+PAS+PTH+SPFX \\
INH+RMP+PZA+SM+Rifa & EMB+PAS+PTH+TZ+SPFX \\
INH+RMP+EMB+Rifa+SMZ & PZA+SM/AM+PTH+PAS+TZ+SPFX \\
INH+RMP+EMB+SM+Rifa & PZA+AM+PTH+PAS+TZ+SPFX \\
INH+RMP+PZA+EMB+SM+Rifa & AM+PTH+PAS+SPFX \\
INH+RMP+PZA+EMB+Rifa+CS & SM+PTH+PAS+CLO+SPFX \\
INH+RMP+PZA+EMB+SM+Rifa+CS+SMZ & PAS+PTH+TZ+AM+SPFX \\
INH+RMP+EMB+SM+Rifa+CS+SMZ+CLM & PZA+PTH+PAS+AM+CLO+SPFX \\
INH+RMP+PZA+EMB+SM+Rifa+PTH+AM & PTH+PAS+TZ+SMZ+SPFX \\
\hline
\end{tabular}

*: patient with history of multi-drug resistant tuberculosis; CI: contraindication; INT: intolerance; INH: isoniazid; RMP: rifampicin; PZA: pyrazinamide; SM: streptomycin; EMB: ethambutol; PAS: para-aminosalicylic acid; PTH: prothionamide; TZ: terizidon; CS: cycloserin; Rifa: rifabutine; AM: amikacin; SPFX: sparfloxacin; CLM: clarithromycin; CLO: clofazimin; SMZ: Sulfamethoxazole.

16 and 18 weeks, respectively, after initiation of therapy. The duration of sparfloxacin treatment during hospitalization was 2.5 and 3 months, respectively, with a daily dosage of $300 \mathrm{mg}$ (table 5). Both patients had culture conversion during hospitalization and significantly improved chest radiograph findings under therapy. They are now treated on an outpatient basis, and a final assessment of treatment outcome is not possible at this time.

\section{Side effects}

In the whole patient group, six moderate prolongations of the electrocardiographic QT interval (30-40 $\mathrm{ms}$ prolongation compared to baseline; $<450 \mathrm{~ms}$ ) were registered without clinical symptoms. In one case, the sparfloxacin dosage had to be reduced from 400 to 200 mg. day $^{-1}$ because of a continuous QT interval prolongation of $40 \mathrm{~ms}$. In all cases with prolonged QT intervals, the follow-up ECGs during therapy returned to normal. Five patients ignored the instruction to avoid sunlight and had mild phototoxic reactions. No case of death was observed, and there was a good hepatic tolerance of sparfloxacin.

\section{Discussion}

The present findings suggest a good therapeutic efficacy of sparfloxacin in the treatment of patients with complicated tuberculosis. In all three groups (resistant/multiresistant, intolerant and continuous proof of $M$. tuberculosis), culture conversion was obtained during hospitalization, and in most cases (24 of 30), baseline chest radiography findings improved significantly. Twenty-five patients completed their regular antituberculosis therapy and could be defined as cured.

Only a limited number of antituberculosis drugs are currently available for MDR TB, none of which are very effective or well tolerated $[2,5,10,13]$. The global increase of MDR TB presents a major treatment problem [2-7]. Due to the high mortality rates associated with MDR TB $(\sim 50 \%)[2,5,6,28]$, it is important to treat these patients with effective therapy regimens of at least three, preferably four, active drugs for a sufficient time period ( 24 months after sputum culture conversion) $[4,29,30]$. In cases of resistance to all first and most or all second line antituberculosis drugs (tables 2 and 3 ), it is very difficult to find an adequate therapy regimen with three or more active drugs. In this study, therapy regimens including

Table 5. - Dosages and durations of sparfloxacin treatment during hospitalization

\begin{tabular}{lccc}
\hline Sparfloxacin dosage & Resistance group & Intolerance group & Continuous proof group \\
\hline Subjects n & 16 & 12 & 2 \\
$400 \mathrm{mg} \cdot \mathrm{day}^{-1}$ & $10^{*}$ & $4^{*}$ & 2 \\
$300 \mathrm{mg} \cdot \mathrm{day}^{-1}$ & $5^{* *}$ & 4 & \\
$200 \mathrm{mg} \cdot$ day $^{-1}$ & 1 & 3 & \\
$100 \mathrm{mg} \cdot$ day $^{-1}$ & & 1 & 2.5 \\
Sparfloxacin therapy duration & 4 & 2.6 & $(3$ months $)$ \\
Mean & $3(1-11$ months $)$ & $2(1-4$ months $)$ & \\
Median (range)
\end{tabular}

*: 1 patient started with $400 \mathrm{mg}$, reduced to $200 \mathrm{mg}$ at the end of therapy; **: 1 patient started with $200 \mathrm{mg}$, subsequently raised to $300 \mathrm{mg}$. 
sparfloxacin yielded excellent results in the treatment of MDR TB. Culture conversion had occurred in all patients by the time of discharge from the hospital, and 10 of 16 patients had significantly improved chest radiograph findings. Thirteen patients completed therapy regularly and could be defined as cured.

The second major problem in the treatment of TB is patients with severe intolerance reactions to first line antituberculosis drugs [8,9]. The most common side effect of antituberculosis therapy is hepatotoxicity, followed by skin reactions [8,9]. The three most important drugs (INH, RMP, PZA) are hepatotoxic. Discontinuation of at least one of three standard drugs due to side effects is necessary in up to $26 \%$ of cases [9]. Risk factors for hepatotoxicity are age $\geqslant 60 \mathrm{yrs}$, history of hepatitis, concomitant intake of other hepatotoxic drugs, diabetes mellitus and alcohol and i.v. drug abuse $[8,9]$. As patients with intolerance also require effective combination therapy with at least three active drugs [29], sparfloxacin may be administered alternatively in these cases.

The intolerance group in the present study also received a therapy regimen including sparfloxacin, with very good results: all patients finished their therapy and could be defined as cured. Sparfloxacin was generally well tolerated. However, mild phototoxic reactions were observed in five cases where the instruction to avoid sunlight had been ignored. Sparfloxacin has a higher phototoxic potential compared to other fluoroquinolones (e.g. ciprofloxacin or levofloxacin) [12]. Patients with MDR TB, proof of $M$. tuberculosis in sputum smear, severe intolerance or other problematic diseases (like alcohol abuse or diabetes mellitus) should be hospitalized [30], and only this patient group should be considered for sparfloxacin therapy. Sunlight exposure is much easier to control when patients are hospitalized. No phototoxic reactions were observed in volunteer studies with sparfloxacin $[17,21,22]$, or in the pneumonia studies $[19,20]$. In these studies, the compliance of volunteers and patients was excellent in contrast to TB patients.

The other problematic side effect of sparfloxacin is the possibility of QT-interval prolongation [12, 15, 18], which was registered in the present study in 6 cases under sparfloxacin therapy (QT-interval prolongation of $30-40 \mathrm{~ms}$ compared to baseline, $<450 \mathrm{~ms}$ ) without clinical symptoms. The daily sparfloxacin dose was reduced in one case, and follow up ECGs of all patients were normal during therapy. A dosage reduction should be considered in all cases with QT-interval prolongation and continuous prolongation in the control ECG. Drugs with a potential of QT-interval prolongation, such as class Ia and III antiarrhythmic agents (e.g. aminodarone, sotalol, quinidine) should be avoided during sparfloxacin therapy $[12,15,18]$, and ECG controls are obligatory.

In conclusion, sparfloxacin is an effective and safe alternative agent in the treatment of complicated tuberculosis and the only suitable alternative drug at present for some cases of multidrug-resistant tuberculosis.

\section{References}

1. WHO. Treatment of tuberculosis. Guidelines for national programmes, WHO/TB 97, 220 Geneva, (1997) 1-77.

2. Cole ST, Telenti A. Drug resistance in Mycobacterium tuberculosis. Eur Respir J 1995; 8: Suppl. 20, 701s$713 \mathrm{~s}$.

3. Hauer B, Loddenkemper R. Tuberkulose: Epidemiologische Entwicklung und Resistenzsituation. DMW 1999; 10: A7-A8.

4. Iseman MD. Treatment of multidrug-resistant tuberculosis. N Engl J Med 1993; 329: 784-791.

5. Reichman LB. Multidrug resistance in the world: The present situation. Chemotherapy 1996; 42: (Suppl. 3), 2-9.

6. Riley LW. Drug-resistant tuberculosis. Clin Infect Dis 1993; 17: (Suppl. 2), S442-446.

7. Schaberg T, Gloger G, Reichert B, Mauch H, Lode H. Drug-resistant pulmonary tuberculosis in Berlin, Germany, 1987-1993. Eur Respir J 1995; 8: 278-284.

8. Ormerod LP, Skinner C, Wales J. Hepatotoxicity of antituberculosis drugs. Thorax 1996; 51: 111-113.

9. Schaberg T, Rebhan K, Lode H. Risk factors for sideeffects of isoniazid, rifampin and pyrazinamide in patients hospitalized for pulmonary tuberculosis. Eur Respir J 1996; 9: 2026-2030.

10. Albino JA, Reichman LB. The treatment of tuberculosis. Respiration 1998; 65: 237-255.

11. O'Brien RJ, Vernon M. New tuberculosis drug development. Am J Respir Crit Care Med 1998; 157: 17051707.

12. Goa KL, Bryson HM, Markham A. Sparfloxacin. Drugs 1997; 53: 700-725.

13. Lalande V, Truffot-Pernot C, Paccaly-Moulin A, Grosset J, Ji B. Powerful bacterial activity of sparfloxacin (AT-4140) against Mycobacterium tuberculosis in mice. Antimicrob Agents Chemother 1993; 37: 407-413.

14. Yew WW, Piddock LJV, Li MSK, Lyon D, Chan CY, Cheng AFB. In-vitro activity of quinolones and macrolides against mycobacteria. $J$ Antimicrob Chemother 1994; 34: 343-351.

15. Carbon C, Rubinstein E. Sparfloxacin monograph. Adis International 1994.

16. Shimada J, Nogita T, Ishibashi Y. Clinical pharmacokinetics of sparfloxacin. Clin Pharmacokinet 1993; 25: 358-369.

17. Schüler P, Zemper K, Borner K, Koeppe P, Schaberg $\mathrm{T}$, Lode H. Penetration of sparfloxacin and ciprofloxacin into alveolar macrophages, epithelial lining fluid, and polymorphonuclear leucocytes. Eur Respir J 1997; 10: 1130-1136.

18. Jaillon P, Morganroth J, Brumpt I, Talbot G, the sparfloxacin safety group. Overview of electrocardiographic and cardiovascular safety data for sparfloxacin. Antimicrob Agents Chemother 1996; 37: (Suppl. A), 161-167.

19. Lode H, Garau J, Grassi C, et al. Treatment of community-acquired pneumonia: a randomized comparison of sparfloxacin, amoxycillin-clavulanic acid and erythromycin. Eur Respir J 1995; 8: 1999-2007.

20. Lode H, Aubier M, Protier H, Ortquist A, the sparfloxacin study group. Sparfloxacin as alternative treatment to standard therapy for communityacquired bacteremic pneumococcal pneumonia. Clin Microbiology Infect 1998; 4: 135-143. 
21. Ritz M, Lode H, Fassbender M, Borner K, Koeppe P, Nord CE. Multiple-dose pharmacokinetics of sparfloxacin and its influence on fecal flora. Antimicrob Agents Chemother 1994; 38: 455-459.

22. Zix JA, Geerdes-Fenge HF, Rau M, et al. Pharmacokinetics of sparfloxacin and interaction with cisapride and sucralfate. Antimicrob Agents Chemother 1997; 41: 1668-1672.

23. WHO. Anti-tuberculosis drug resistance in the world. The WHO-IUATLD Global project on antituberculosis drug resistance surveillance. WHO/TB/97. 229: 36.

24. Veen J, Raviglione M, Rieder HL, et al. Standardized tuberculosis treatment outcome monitoring in Europe. Eur Respir J 1998; 12: 505-510.

25. American Thoracic Society. Diagnostic standards and classification of tuberculosis. Am Rev Respir Dis 1990; 142: 725-735.

26. Kent TP, Kubica GP. A guide for the level III laboratory. Atlanta; Centres for Disease Control, 1985.

27. Deutsches Zentralkomitee zur Bekämpfung der Tuberkulose. Die Bakteriologie der Tuberculose. Pneumologie 1991; 45: 753-774.

28. Turett GS, Telzak EE, Torian LV. Improved outcomes for patients with multidrug resistant tuberculosis. Clin Infect Dis 1995; 21: 1238-1244.

29. American Thoracic Society. Treatment of tuberculosis and tuberculosis infection in adults and children. $\mathrm{Am} \mathrm{J}$ Respir Crit Care Med 1994; 149: 1359-1374.

30. Deutsches Zentralkomitee zur Bekämpfung der Tuberkulose. Richtlinien zur Chemotherapie der Tuberkulose. Pneumologie 1995; 49: 217-225. 